\title{
Gérard Khan, Beaumarchais. Le Mariage de Figaro
}

\section{Claudio Vinti}

\section{(2) OpenEdition}

\section{Journals}

\section{Edizione digitale}

URL: https://journals.openedition.org/studifrancesi/39477

DOI: 10.4000/studifrancesi.39477

ISSN: 2421-5856

\section{Editore}

Rosenberg \& Sellier

\section{Edizione cartacea}

Data di pubblicazione: 1 décembre 2004

Paginazione: $379-380$

ISSN: 0039-2944

\section{Notizia bibliografica digitale}

Claudio Vinti, "Gérard Khan, Beaumarchais. Le Mariage de Figaro», Studi Francesi [Online], 143 (XLVIII ]

II) | 2004, online dal 30 novembre 2015, consultato il 19 mai 2021. URL: http://

journals.openedition.org/studifrancesi/39477 ; DOI: https://doi.org/10.4000/studifrancesi.39477

Questo documento è stato generato automaticamente il 19 mai 2021.

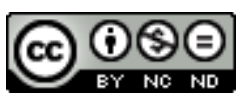

Studi Francesi è distribuita con Licenza Creative Commons Attribuzione - Non commerciale - Non opere derivate 4.0 Internazionale. 


\title{
Gérard Khan, Beaumarchais. Le Mariage de Figaro
}

\author{
Claudio Vinti
}

\section{NOTIZIA}

GÉRARD KHAN, Beaumarchais. Le Mariage de Figaro. Edition critique avec les variantes de la version scenique originale, Oxford, Voltaire Foundation («Studies on Voltaire and the Eighteenth Century»), 2002, pp. 520.

1 Questo volume è il frutto di un immane lavoro di ricerca sui manoscritti e sulle prime edizioni a stampa del Mariage, nel tentativo di ricostruire il più fedelmente possibile la prima versione scenica della pièce. Con questo lavoro l'A. ha cercato di capire perché Beaumarchais nella sua edizione autorizzata della pièce non abbia utilizzato il testo della prima rappresentazione e si sia invece attenuto al manoscritto della Comédie Française, tra l'altro anteriore alla rappresentazione stessa. Ancora una volta, come sempre accade nel teatro, il problema fondamentale che si pone è la differenza tra testo scritto e testo rappresentato. Gérard Khan fa varie ipotesi per spiegare le sostanziali differenze tra questi due testi, ipotesi che vanno dalla presunta volontà di Beaumarchais di prendere le distanze dalla versione dei Comédiens, al rispetto della proprietà letteraria, come se la versione scenica non fosse interamente di sua proprietà, fino alla inopportunità di dare alle stampe un testo preparato per essere visto e non per essere letto. Quali che siano state le ragioni che hanno spinto Beaumarchais ad attenersi al manoscritto della Comédie Française, le varianti rilevate da Gerard Khan fra il testo rappresentato e la versione autorizzata, permettono all'A. di evidenziare alcuni tratti caratteristici della versione scenica, che rendono il testo più fluido, grazie all'aggiunta di articolatori, più vivace, grazie all'aggiunta di interiezioni, più limpido sintatticamente, più trasparente, moderno e adattabile al grande pubblico, grazie all'eliminazione di arcaismi. Il testo della versione scenica, inoltre, rivela una lingua più adeguata al rango dei personaggi, più rispettosa delle «bienséances», con l'eliminazione di passaggi sconvenienti o troppo arditi. II risultato è una versione del 
Mariage de Figaro più gradevole e più facile da seguire. Tutti questi elementi prosodici e visivi saranno minuziosamente descritti da Beaumarchais nelle didascalie della versione autorizzata. Didascalie che ci forniscono una infinità di informazioni supplementari, relative all'intonazione, al ritmo (in particolare alle pause), agli accessori e ai costumi di scena, alla mimica. E tuttavia nella registrazione delle componenti essenziali del mestiere di attore che si evidenzia l'importanza delle didascalie. Se è vero che a teatro linguaggio verbale e linguaggio non verbale hanno un ruolo quasi pari, nel Mariage de Figaro, al dialogo spumeggiante, che è proprio della pièce, fanno da contrappunto le componenti essenziali del mestiere di attore: mimica del viso, gestualità, movimento sul palcoscenico e tutta quella comunicazione non verbale che, in ultima analisi, è l'essenza dell'arte dell'attore. Lo straordinario assemblaggio proposto da Gerard Khan dà vita a una versione scenica del Mariage estremamente convincente, le cui varianti testuali e mimico-gestuali fanno evolvere la pièce verso un lettura più gioiosa e meno melodrammatica, tendente a lasciare più autonomia all'attore in scena, sulla scia di Diderot e nella migliore tradizione del teatro italiano (sull'importanza della mimica e della gestualità a teatro, si veda anche il recente C. BOURQUI - C. VINTI, Molière à l'École italienne). 